\title{
Brachial Plexopathy from Metaldehyde Poisoning - A Case Report
}

\author{
${ }^{1}$ See-Teng Tan, ${ }^{2}$ Chen Guanglin, ${ }^{1}$ Deanna Lee, ${ }^{3}$ You-Jiang Tan \\ ${ }^{1}$ Department of General Medicine, Sengkang General Hospital, Singapore, ${ }^{2}$ DUKE-NUS Medical \\ School, Singapore, ${ }^{3}$ Department of Neurology, National Neuroscience Institute, Singapore
}

\begin{abstract}
We describe the case of a middle-aged female with schizophrenia, who developed acute sensorimotor deficits of the right upper limb within a week of ingesting large amounts of metaldehyde in a suicide attempt. A right-sided brachial plexopathy was diagnosed clinically and supported by electrophysiologic assessments and targeted magnetic resonance imaging scans. Although metaldehyde's neurotoxicity typically affects the central nervous system, focal involvement of the peripheral nervous system remains unreported in medical literature, and its pathogenic processes await further elucidation. Therefore, we recommend the continued observation for the subsequent development of sensorimotor deficits during the first week of metaldehyde poisoning.
\end{abstract}

Keywords: Metaldehyde, Brachial plexopathy, Neurotoxicity

\section{INTRODUCTION}

Metaldehyde, a cyclic polymer of acetaldehyde, is a commonly-used molluscicide. Classified as a class II toxin by the World Health Organization (WHO), it can cause moderately hazardous acute health effects through its effects on the central nervous system (CNS), liver, kidney, and lungs. (1) Although its toxicity in smaller mammals is well-studied, ingestion at higher doses of $100 \mathrm{mg} / \mathrm{kg}$ can result in hyperthermia, seizures, acid-base imbalances, hepatic dysfunction, and death, toxicity in humans remains uncommon and consequently poorly understood. (2)

Herein, we describe the unique case of a 46-year-old woman who developed a right-sided brachial plexopathy within a week of ingesting metaldehyde whilst attempting suicide. While metaldehyde's toxicity on the CNS of small mammals has been established in preceding literature, its focal effects on the peripheral nervous system resulting in plexopathies have not been reported before.

\section{CASE REPORT}

A 46-year-old woman was found delirious after attempting suicide by ingesting the equivalent of $150 \mathrm{mg} / \mathrm{kg}$ of metaldehyde. She has a history of schizophrenia and was treated with olanzapine. Last observed well in the morning, she was discovered in the afternoon to be lethargic on her bed. She scored 9 on the Glasgow Coma Scale (GCS; E3V4M2) and was hemodynamically stable (blood pressure $107 / 77 \mathrm{mmHg}$, heart rate $96 \mathrm{bpm})$. She had no sensorimotor deficits, and examination of her precordium and abdomen yielded unremarkable findings. She underwent gastric lavage, received oral activated charcoal, and was given fluids intravenously. Her recovery was complicated by high anion-gap metabolic acidosis and rhabdomyolysis, the latter resulting in elevated serum creatine kinase and aldolase levels of 16261U/L (range 44-201U/L) $58.2 \mathrm{U} / \mathrm{L}$ (range 1.3-6.3U/L), respectively. No seizures were observed before and during her hospitalization. Blood and urine toxicology tests demonstrated the presence of olanzapine, but without detection of other substances.

On day 2 of hospitalization, she experienced pain over the right shoulder and sensorimotor deficits of her right upper limb. There was no preceding trauma. Examination revealed monoplegia (Medical Research Council grade 0 ) and anaesthesia to pinprick and fine touch involving the entire right upper limb. Retraction of the scapula remained strong, and scapular winging was absent. Deep tendon reflexes of the right-sided biceps brachii, triceps brachii and brachioradialis muscles were absent. Sensorimotor findings of the other limbs were unremarkable,

Address correspondence to: See Teng Tan, Department of General Medicine, Sengkang General Hospital, 110 Sengkang East Way, Singapore 544886 Email: seeteng.tan@mohh.com.sg

Date of Submission: 31 January 2021; Date of Acceptance: 15 March 2021

https://doi.org/10.54029/2021 wry 
and palpation over the arms' musculature did not elicit pain. Right-sided brachial plexopathy was suspected based on the clinical findings, for which she underwent further electrophysiological, imaging, and immunologic studies. She tested negative for the human immunodeficiency virus, while serum antinuclear and anti-neutrophil cytoplasmic antibody assays returned negative. Magnetic resonance imaging (MRI) of the cervical spine demonstrated multilevel cervical spondylosis with moderately severe bilateral foraminal stenosis worst at C5-6. Performed 2 weeks from symptom-onset, a nerve conduction study (NCS) revealed findings consistent with a right-sided pan-brachial plexopathy (Table 1), but the brachial plexus MRI with gadolinium contrast did not demonstrate abnormalities of both brachial plexuses, showing instead of the asymmetric fatty atrophy, oedema, and enhancement predominantly affecting the rightsided supraspinatus, infraspinatus, subscapularis and teres minor muscles (Figure 1A). Similar but remarkably milder MRI abnormalities were observed over the left-sided muscles (Figure 1B). While muscular fatty atrophy and oedema were expected in view of the rhabdomyolysis, we attributed the remarkable asymmetry to postdenervation changes from the concomitant rightsided brachial plexopathy. Given the temporal relationship of her right upper-limb weakness and the consumption of injurious amounts of metaldehyde, and the absence of clinical and diagnostic abnormalities suggestive of an alternative cause of her brachial plexopathy, she was thus diagnosed with a right-sided pan-brachial plexopathy secondary to metaldehyde toxicity. Despite two months of physiotherapy, the right upper limb remained monoplegic and anaesthetic.

\section{DISCUSSION}

The neurotoxicity of metaldehyde has been previously documented in humans, manifesting primarily as perturbations of the central nervous system (CNS). (2-4) However, focal and lasting insults to the peripheral nervous system, in our case a unilateral brachial plexopathy, has not been previously reported in the literature. The exact mechanism through which metaldehyde exerts its neurotoxic effects in humans is unknown; rodent studies suggest metaldehyde increases monoamine oxidase activity, thus decreasing norepinephrine (NE), serotonin (5-HT) and gamma-aminobutyric acid (GABA) concentrations in the central nervous system. (5) These potentially explain the
CNS manifestations of seizures from increased neuronal excitability in metaldehyde poisoning. Molluscan studies performed in Lymnaea stagnalis have also shown a direct neurotoxic effect of metaldehyde on neural circuitry. By altering membrane conductance by reducing outward potassium currents, metaldehyde increases action potential firing frequency, level of depolarization and induces paroxysmal depolarizing shifts in motor neurons of L. stagnalis, though resulting in muscle spasms. $(6,7)$ Taken together, metaldehyde may thus manifest its neurotoxic effects on both the CNS and peripheral nervous system through neuronal hyperexcitability. However, the focal and lasting nature of insult to our patient's brachial plexus remains difficult to explain with neuronal hyperexcitability alone and will benefit from further illumination by future studies.

Another learning point from our patient is the temporal interval of 2 days between the clinical onset of brachial plexopathy and the ingestion of metaldehyde. CNS manifestations from metaldehyde toxicity tended to be florid and obvious soon after ingestion, likely explained by its neuro-excitatory mechanisms. However, the interlude of sensorimotor normalcy observed in this case again casts doubt on metaldehydeinduced neuronal hyperexcitability being the primary mechanism behind her brachial plexopathy. Regardless, it seems reasonable to recommend continued observation for the subsequent development of sensorimotor deficits during the first week of metaldehyde poisoning.

\section{CONCLUSIONS}

To the best of our knowledge, this is the first reported case of brachial plexopathy caused by metaldehyde poisoning and introduces a new neurological complication in humans. Although CNS manifestations have been theorized to be due to metaldehyde-induced neuronal hyperexcitability, the focal and lasting involvement of our patient's peripheral nervous system suggests a separate pathogenic process. Additionally, physicians should monitor the patient for subsequent sensorimotor deficits, at least for the few days after metaldehyde poisoning. 
Table 1. Nerve conduction study results Motor Study

\begin{tabular}{|c|c|c|c|c|}
\hline Stimulation site & Latency (ms) & Amplitude (mV) & $\begin{array}{l}\text { Conduction velocity } \\
(\mathrm{m} / \mathrm{s})\end{array}$ & $\begin{array}{l}\text { F minimum latency } \\
(\mathrm{ms})\end{array}$ \\
\hline \multicolumn{5}{|c|}{ Right median nerve (abductor pollicis brevis) } \\
\hline Wrist & 2.7 & 5.4 & 57 & 27.56 \\
\hline Elbow & 6.4 & 5.4 & 63 & \\
\hline Erb's point & 11.7 & 3.1 & & \\
\hline \multicolumn{5}{|c|}{ Left median nerve (abductor pollicis brevis) } \\
\hline Wrist & 2.7 & 17.3 & 58 & 24.02 \\
\hline Elbow & 6.4 & 16.8 & 68 & \\
\hline Erb's point & 11.6 & 14.7 & & \\
\hline \multicolumn{5}{|c|}{ Right ulnar nerve (abductor digiti minimi) } \\
\hline Wrist & 2.0 & 4.1 & 57 & 28.83 \\
\hline Below elbow & 3.6 & 4.1 & 50 & \\
\hline Above elbow & 6.6 & 3.9 & 70 & \\
\hline Erb's point & 11.3 & 3.4 & & \\
\hline \multicolumn{5}{|c|}{ Left ulnar nerve (abductor digiti minimi) } \\
\hline Wrist & 2.3 & 9.4 & 63 & 24.16 \\
\hline Below elbow & 3.8 & 9.3 & 60 & \\
\hline Above elbow & 6.6 & 9.1 & 74 & \\
\hline Erb's point & 11.1 & 8.4 & & \\
\hline
\end{tabular}

Right radial nerve (extensor indicis proprius)

\begin{tabular}{llll}
\hline Elbow & 2.4 & 2.6 & 60 \\
\hline Spiral groove & 5.0 & 2.4 & 88 \\
\hline Erb's point & 8.2 & 1.8 & \\
\hline
\end{tabular}

Left radial nerve (extensor indicis proprius)

\begin{tabular}{lllc}
\hline Elbow & 2.5 & 5.7 & 56 \\
\hline Spiral groove & 5.0 & 5.0 & 129 \\
\hline Erb's point & 7.2 & 4.6 & \\
\hline
\end{tabular}

Right axillary nerve (deltoid)

\begin{tabular}{lll}
\hline Erb's point & 3.7 & 0.6
\end{tabular}

Left axillary nerve (deltoid)

\begin{tabular}{lll}
\hline Erb's point & 3.2 & 9.4
\end{tabular}

Right musculocutaneous nerve (biceps brachii)

\begin{tabular}{lll}
\hline Erb's point & 3.8 & 0.7
\end{tabular}

Right musculocutaneous nerve (biceps brachii)

\begin{tabular}{lll}
\hline Erb's point & 3.1 & 12.8
\end{tabular}

Second lumbrical-interosseous ( $2^{\text {nd }}$ lumbrical)

\begin{tabular}{lll}
\hline Right median nerve & 3.0 & 0.7 \\
\hline Right ulnar nerve & 2.6 & 2.2 \\
\hline Left median nerve & 3.0 & 2.1 \\
\hline Left ulnar nerve & 2.7 & 5.8
\end{tabular}


Sensory Study

\begin{tabular}{|c|c|c|c|}
\hline Stimulation site & Latency (ms) & Amplitude (mV) & Conduction velocity $(\mathrm{m} / \mathrm{s})$ \\
\hline \multicolumn{4}{|c|}{ Right median nerve (Digit II) } \\
\hline & 2.3 & 8.6 & 57 \\
\hline \multicolumn{4}{|c|}{ Left median nerve (Digit II) } \\
\hline & 2.2 & 73.1 & 59 \\
\hline \multicolumn{4}{|c|}{ Right ulnar nerve (Digit V) } \\
\hline & 1.9 & 20.0 & 58 \\
\hline \multicolumn{4}{|c|}{ Left ulnar nerve (Digit V) } \\
\hline & 2.0 & 68.8 & 55 \\
\hline \multicolumn{4}{|c|}{ Right radial nerve (Base of thumb) } \\
\hline & 1.8 & 2.3 & 53 \\
\hline \multicolumn{4}{|c|}{ Left radial nerve (Base of thumb) } \\
\hline & 1.8 & 28.3 & 57 \\
\hline \multicolumn{4}{|c|}{ Right lateral antebrachial cutaneous sensory nerve (Lateral forearm) } \\
\hline & 2.3 & 3.3 & 53 \\
\hline \multicolumn{4}{|c|}{ Left lateral antebrachial cutaneous sensory nerve (Lateral forearm) } \\
\hline & 1.9 & 41.7 & 64 \\
\hline \multicolumn{4}{|c|}{ Right medial antebrachial cutaneous sensory nerve (Medial forearm) } \\
\hline & 2.2 & 3.1 & 58 \\
\hline \multicolumn{4}{|c|}{ Left medial antebrachial cutaneous sensory nerve (Medial forearm) } \\
\hline & 2.2 & 17.9 & 59 \\
\hline
\end{tabular}

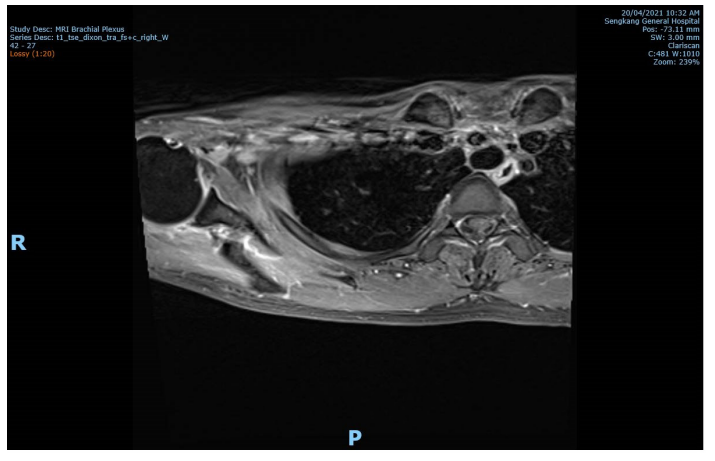

Figure 1A. Asymmetric fatty atrophy, oedema, and enhancement predominantly affecting the right-sided supraspinatus, infraspinatus, subscapularis and teres minor muscles.

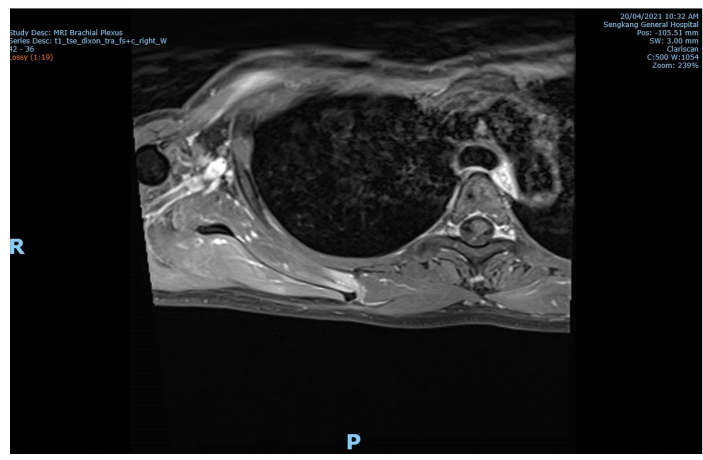

Figure 2A. Axial T1-weighted MR image obtained at the level of the T1 vertebral body shows the anatomy of the right brachial plexus.

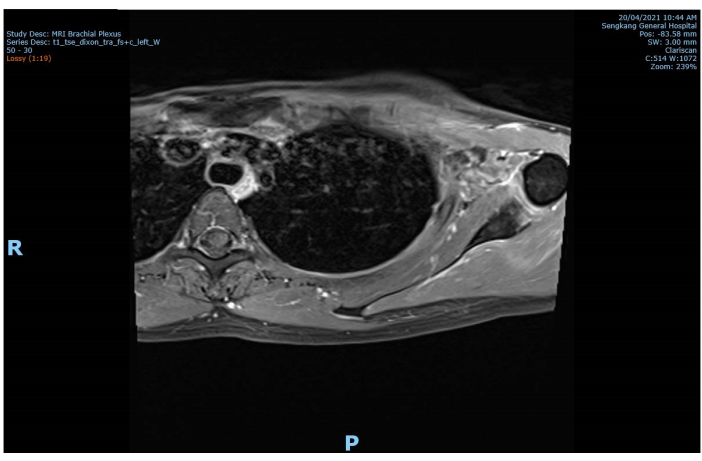

Figure 1B. Asymmetric fatty atrophy, oedema, and enhancement predominantly affecting the right-sided supraspinatus, infraspinatus, subscapularis and teres minor muscles.

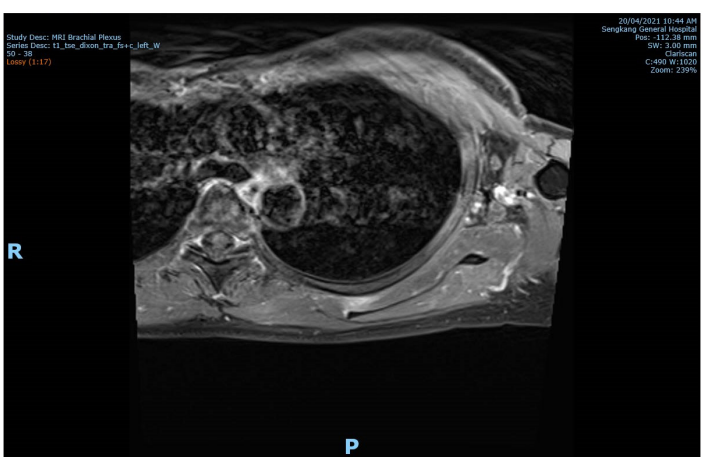

Figure 2B. Axial T1-weighted MR image obtained at the level of the T1 vertebral body shows the anatomy of the left brachial plexus. 


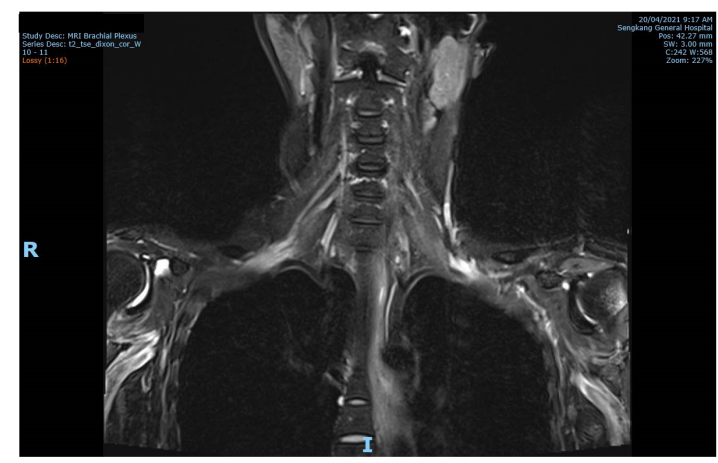

Figure 3. MRI Brachial plexus demonstrating the left and right asymmetric denervation changes (Coronol Projection).

\section{Author Declaration}

Any opinions, findings, conclusions, or recommendations expressed in this material are those of the authors and do not reflect the views of the Ministry of Health or the SingHealth institution.

All authors disclosed no financial associations or other possible conflicts of interests.

\section{Ethics}

This study has been granted exemption by the SINGHEALTH CENTRALISED INSTITUTIONAL REVIEW BOARD (CIRB) DETERMINATION.

\section{REFERENCES}

1. WHO Recommended Classification of Pesticides by Hazard and Guidelines to Classification 2009 WHOSG2. WHO Recommended Classification of Pesticides by Hazard and Guidelines to Classification 2009. In. Stuttgart: World Health Organization; 2010.

2. Fernández-Torre JL, Paramio-Paz A, RodríguezBorregán JC, et al. Super-refractory nonconvulsive status epilepticus due to self-poisoning with metaldehyde. J of Clin Neurosci 2018 Jan;47:134136.

3. Shih CC, Chang SS, Chan YL, et al. Acute metaldehyde poisoning in Taiwan. Vet Hum Toxicol. 2004 Jun;46(3):140-3.

4. Longstreth WT Jr, \& Pierson DJ. Metaldehyde poisoning from slug bait ingestion. West J Med. 1982;137(2):134-137

5. Sparks S, Quistad G, Cole L, et al.(1996). Metaldehyde Molluscicide Action in Mice: Distribution, Metabolism, and Possible Relation to GABAergic System. Pestic Biochem Physiol, 1996:55(3), 226236.

6. Mills, JD, McCrohan CR, Bailey SE. Electrophysiological responses to metaldehyde in neurones of the feeding circuitry of the snail Lymnaea stagnalis. Pestic Biochem Physiol. 1992:42(1):35-42 